Supporting Information for:

\title{
Scaffold-Based Functional Models of \\ [Fe]-Hydrogenase (Hmd): Building the Bridge between Biological Structure and Molecular Function
}

\author{
Spencer A. Kerns and Michael J. Rose* \\ Department of Chemistry, The University of Texas at Austin \\ Austin, Texas 78712, United States
}

\section{Computational Details for Organic Substrates}

The computational methods described by Glusac and coworkers were used to calculate the hydricities of ${ }^{\mathrm{To}} \mathrm{ImH}$ and ${ }^{\mathrm{Me}} \mathrm{mmH}$ presented in the manuscript. All calculations were performed using the Gaussian 09 package. ${ }^{1}$ The structures ${ }^{\mathrm{Tol}} \mathrm{ImH}, \mathrm{Me}^{\mathrm{M}} \mathrm{mH},{ }^{\mathrm{T} o l} / \mathrm{m}^{+}$, and $\mathrm{Me} / \mathrm{m}^{+}$were optimized at the wB97X-D/6-311G(d) level of theory with the CPCM for acetonitrile. Upon geometric optimization, a frequency calculation was performed to ensure that there were no imaginary frequencies. A single-point energy calculation was then performed with wB97X-D/6-311G++(2df,p) with the CPCM for acetonitrile. Further computational details and a detailed description of the calculation of hydricity can be found at the provided reference. ${ }^{2}$

\section{DFT Geometry Optimized Coordinates of Substrates:}

\section{Tol/mH}

Energy: -852079.8758530

$\begin{array}{lrrr}\text { N } & -1.27208 & -1.00352 & -0.66555 \\ \mathrm{~N} & 0.91387 & -1.47155 & -0.28072 \\ \mathrm{~F} & -2.50270 & -2.16443 & 1.58796 \\ \mathrm{~F} & -2.78599 & 0.85169 & -2.00020 \\ \mathrm{~F} & 1.76331 & -1.10466 & 2.29960 \\ \mathrm{~F} & 2.90072 & -1.15696 & -2.27110 \\ \mathrm{C} & -0.17766 & -0.63801 & 0.23452 \\ \mathrm{H} & -0.39810 & -0.91929 & 1.27250 \\ \mathrm{C} & -1.02060 & -2.35557 & -1.15505 \\ \mathrm{H} & -1.59224 & -2.54172 & -2.06523 \\ \mathrm{H} & -1.25916 & -3.13021 & -0.41422 \\ \mathrm{C} & 0.47427 & -2.28340 & -1.41056 \\ \mathrm{H} & 0.95657 & -3.26287 & -1.39021 \\ \mathrm{H} & 0.66931 & -1.81355 & -2.37829 \\ \mathrm{C} & 0.11099 & 0.84613 & 0.19000 \\ \mathrm{C} & 0.07188 & 1.60889 & 1.34784 \\ \mathrm{H} & -0.17072 & 1.13496 & 2.29454 \\ \mathrm{C} & 0.34714 & 2.97444 & 1.30682 \\ \mathrm{H} & 0.30739 & 3.55493 & 2.22398\end{array}$




$\begin{array}{lrrr}\mathrm{C} & 0.67398 & 3.60102 & 0.10832 \\ \mathrm{C} & 0.71489 & 2.82203 & -1.05481 \\ \mathrm{H} & 0.96519 & 3.29220 & -2.00178 \\ \mathrm{C} & 0.43700 & 1.46664 & -1.01621 \\ \mathrm{H} & 0.46464 & 0.87697 & -1.92680 \\ \mathrm{C} & 0.99452 & 5.07145 & 0.05265 \\ \mathrm{H} & 0.74444 & 5.57221 & 0.98988 \\ \mathrm{H} & 0.44708 & 5.56642 & -0.75378 \\ \mathrm{H} & 2.06053 & 5.23140 & -0.13577 \\ \mathrm{C} & -2.57237 & -0.67270 & -0.22627 \\ \mathrm{C} & -3.18808 & -1.23849 & 0.89210 \\ \mathrm{C} & -4.45679 & -0.89361 & 1.31580 \\ \mathrm{H} & -4.87749 & -1.36970 & 2.19292 \\ \mathrm{C} & -5.15881 & 0.06384 & 0.59311 \\ \mathrm{H} & -6.15576 & 0.34834 & 0.90807 \\ \mathrm{C} & -4.59653 & 0.65771 & -0.52886 \\ \mathrm{H} & -5.12993 & 1.40372 & -1.10505 \\ \mathrm{C} & -3.32210 & 0.27999 & -0.91489 \\ \mathrm{C} & 2.23810 & -1.16931 & -0.00437 \\ \mathrm{C} & 3.23927 & -1.02945 & -0.97196 \\ \mathrm{C} & 4.56014 & -0.75407 & -0.67134 \\ \mathrm{H} & 5.27602 & -0.67114 & -1.48026 \\ \mathrm{C} & 4.92743 & -0.55486 & 0.65232 \\ \mathrm{H} & 5.95548 & -0.32197 & 0.90112 \\ \mathrm{C} & 3.97111 & -0.65391 & 1.65538 \\ \mathrm{H} & 4.22024 & -0.51055 & 2.69985 \\ \mathrm{C} & 2.67321 & -0.97302 & 1.31320\end{array}$

Me ImH

Energy: -707049.6258129

$\begin{array}{lrrr}\mathrm{N} & 1.12238 & -0.62320 & -0.32012 \\ \mathrm{~N} & -1.10658 & -0.22002 & -0.53851 \\ \mathrm{~F} & 2.14692 & 1.78896 & -1.36653 \\ \mathrm{~F} & 2.87069 & -2.06730 & 1.21148 \\ \mathrm{~F} & -1.88043 & 2.28753 & 0.28746 \\ \mathrm{~F} & -3.14659 & -2.18595 & -0.50467 \\ \mathrm{C} & 0.04437 & 0.16428 & 0.28806 \\ \mathrm{H} & 0.22875 & 1.23995 & 0.17873 \\ \mathrm{C} & 0.73798 & -0.96054 & -1.68732 \\ \mathrm{H} & 1.29914 & -1.82928 & -2.03529 \\ \mathrm{H} & 0.88345 & -0.13212 & -2.39331 \\ \mathrm{C} & -0.73643 & -1.25774 & -1.49431 \\ \mathrm{H} & -1.31412 & -1.15681 & -2.41503 \\ \mathrm{H} & -0.86963 & -2.26962 & -1.10103 \\ \mathrm{C} & -0.10917 & -0.17362 & 1.76221\end{array}$




$\begin{array}{lrrr}\mathrm{C} & 2.43894 & -0.16580 & -0.09538 \\ \mathrm{C} & 2.95714 & 1.02382 & -0.61260 \\ \mathrm{C} & 4.25197 & 1.44929 & -0.38688 \\ \mathrm{H} & 4.59306 & 2.38187 & -0.81930 \\ \mathrm{C} & 5.08308 & 0.65674 & 0.39620 \\ \mathrm{H} & 6.10237 & 0.97204 & 0.58481 \\ \mathrm{C} & 4.62057 & -0.53463 & 0.93884 \\ \mathrm{H} & 5.25324 & -1.16403 & 1.55243 \\ \mathrm{C} & 3.31589 & -0.91930 & 0.68384 \\ \mathrm{C} & -2.41033 & 0.03356 & -0.14673 \\ \mathrm{C} & -3.43962 & -0.91727 & -0.15116 \\ \mathrm{C} & -4.74807 & -0.63265 & 0.19214 \\ \mathrm{H} & -5.48325 & -1.42742 & 0.15179 \\ \mathrm{C} & -5.07916 & 0.64770 & 0.61208 \\ \mathrm{H} & -6.09722 & 0.88020 & 0.89917 \\ \mathrm{C} & -4.09633 & 1.62840 & 0.66191 \\ \mathrm{H} & -4.31449 & 2.64217 & 0.97550 \\ \mathrm{C} & -2.81223 & 1.31175 & 0.26977 \\ \mathrm{H} & -0.90455 & 0.41728 & 2.22270 \\ \mathrm{H} & -0.33419 & -1.23576 & 1.88307 \\ \mathrm{H} & 0.82004 & 0.05113 & 2.29258\end{array}$

Tol $/ m^{+}$

Energy: -851506.1443471

$\begin{array}{lrrr}\text { N } & -1.09546 & -1.39889 & 0.08700 \\ \text { N } & 1.08684 & -1.40359 & -0.08540 \\ \mathrm{~F} & -2.89661 & -1.49710 & 2.14855 \\ \mathrm{~F} & -1.98098 & -0.30003 & -2.27235 \\ \mathrm{~F} & 1.97666 & -0.30438 & 2.27227 \\ \mathrm{~F} & 2.88766 & -1.51233 & -2.14664 \\ \mathrm{C} & -0.00278 & -0.65571 & -0.00015 \\ \mathrm{C} & -0.77544 & -2.83928 & 0.03030 \\ \mathrm{H} & -1.22877 & -3.26561 & -0.86368 \\ \mathrm{H} & -1.17116 & -3.33885 & 0.91240 \\ \mathrm{C} & 0.76107 & -2.84252 & -0.02485 \\ \mathrm{H} & 1.21261 & -3.26831 & 0.87030 \\ \mathrm{H} & 1.15478 & -3.34606 & -0.90558 \\ \mathrm{C} & 0.00076 & 0.80658 & -0.00127 \\ \mathrm{C} & -0.67013 & 1.50225 & 1.00311 \\ \mathrm{H} & -1.17840 & 0.96636 & 1.79649 \\ \mathrm{C} & -0.65570 & 2.88775 & 0.99969 \\ \mathrm{H} & -1.16918 & 3.42425 & 1.79026 \\ \mathrm{C} & 0.00670 & 3.59800 & -0.00282 \\ \mathrm{C} & 0.66456 & 2.88266 & -1.00744 \\ \mathrm{H} & 1.17511 & 3.41733 & -1.80143\end{array}$




$\begin{array}{lrrr}\mathrm{C} & 0.67423 & 1.49888 & -1.00859 \\ \mathrm{H} & 1.17675 & 0.95923 & -1.80311 \\ \mathrm{C} & 0.03570 & 5.10069 & 0.00431 \\ \mathrm{H} & -0.74055 & 5.51330 & 0.65032 \\ \mathrm{H} & -0.10015 & 5.50472 & -1.00105 \\ \mathrm{H} & 1.00135 & 5.46028 & 0.37236 \\ \mathrm{C} & -2.41556 & -0.91057 & -0.05924 \\ \mathrm{C} & -3.32287 & -0.98218 & 0.99128 \\ \mathrm{C} & -4.61885 & -0.52375 & 0.86760 \\ \mathrm{H} & -5.29471 & -0.59186 & 1.71036 \\ \mathrm{C} & -5.01458 & 0.02751 & -0.34559 \\ \mathrm{H} & -6.02623 & 0.39823 & -0.45573 \\ \mathrm{C} & -4.13682 & 0.11579 & -1.41980 \\ \mathrm{H} & -4.43615 & 0.54368 & -2.36790 \\ \mathrm{C} & -2.85268 & -0.36367 & -1.26165 \\ \mathrm{C} & 2.40876 & -0.91998 & 0.06003 \\ \mathrm{C} & 2.84797 & -0.37283 & 1.26158 \\ \mathrm{C} & 4.13399 & 0.10184 & 1.41898 \\ \mathrm{H} & 4.43503 & 0.53004 & 2.36641 \\ \mathrm{C} & 5.01146 & 0.00828 & 0.34498 \\ \mathrm{H} & 6.02462 & 0.37499 & 0.45460 \\ \mathrm{C} & 4.61362 & -0.54346 & -0.86731 \\ \mathrm{H} & 5.28930 & -0.61581 & -1.70985 \\ \mathrm{C} & 3.31583 & -0.99692 & -0.99032\end{array}$

$\mathrm{Me} / \mathrm{m}^{+}$

Energy: -706590.5553786

$\begin{array}{lrrr}\mathrm{N} & -1.09119 & 0.33738 & 0.48833 \\ \mathrm{~N} & 1.09066 & 0.33330 & 0.49265 \\ \mathrm{~F} & -2.76453 & 2.33374 & -0.35675 \\ \mathrm{~F} & -2.12523 & -2.21196 & 0.51044 \\ \mathrm{~F} & 2.75350 & 2.33068 & -0.37939 \\ \mathrm{~F} & 2.13478 & -2.20904 & 0.53421 \\ \mathrm{C} & 0.00047 & -0.01039 & -0.17139 \\ \mathrm{C} & -0.76928 & 0.96900 & 1.78293 \\ \mathrm{H} & -1.16280 & 0.35056 & 2.58885 \\ \mathrm{H} & -1.22032 & 1.95809 & 1.83049 \\ \mathrm{C} & 0.76679 & 1.01513 & 1.76080 \\ \mathrm{H} & 1.15866 & 2.03057 & 1.73495 \\ \mathrm{H} & 1.21896 & 0.47208 & 2.58892 \\ \mathrm{C} & 0.00372 & -0.71336 & -1.47185 \\ \mathrm{C} & -2.41858 & 0.06620 & 0.08444 \\ \mathrm{C} & -3.26214 & 1.09493 & -0.32365 \\ \mathrm{C} & -4.57369 & 0.86202 & -0.68454 \\ \mathrm{H} & -5.19876 & 1.68880 & -0.99669\end{array}$




$\begin{array}{lrrr}\mathrm{C} & -5.05231 & -0.44239 & -0.64094 \\ \mathrm{H} & -6.07910 & -0.64007 & -0.92302 \\ \mathrm{C} & -4.24056 & -1.49785 & -0.24231 \\ \mathrm{H} & -4.60561 & -2.51615 & -0.20504 \\ \mathrm{C} & -2.93727 & -1.22453 & 0.12068 \\ \mathrm{C} & 2.41825 & 0.06646 & 0.08701 \\ \mathrm{C} & 3.25673 & 1.09430 & -0.33351 \\ \mathrm{C} & 4.56858 & 0.86323 & -0.69438 \\ \mathrm{H} & 5.18967 & 1.68947 & -1.01575 \\ \mathrm{C} & 5.05260 & -0.43875 & -0.63891 \\ \mathrm{H} & 6.07972 & -0.63505 & -0.92074 \\ \mathrm{C} & 4.24561 & -1.49351 & -0.22906 \\ \mathrm{H} & 4.61475 & -2.50996 & -0.18260 \\ \mathrm{C} & 2.94192 & -1.22187 & 0.13392 \\ \mathrm{H} & 0.03137 & -1.79043 & -1.29155 \\ \mathrm{H} & -0.89755 & -0.47819 & -2.03521 \\ \mathrm{H} & 0.88287 & -0.43697 & -2.05161\end{array}$

\section{DFT on Metal Complexes}

Geometry optimizations for the structures of $\mathbf{1}^{+}$and $\mathbf{5}^{+}$were performed with the Firefly ${ }^{3}$ software package with combinations of the $6-31 \mathrm{G}(\mathrm{d})$-combo and the functional PW91 ${ }^{4}$. The optimized structures of $\mathbf{1}^{+}$and $\mathbf{5}^{+}$were based on closely related crystal structures. In the case of $1^{+}$, a phosphine bound complex (with thioether-S displaced) was used as a starting coordinates and modified to include bound thioether-S and open sixth coordination site. In the case of $5^{+}$, the bromide bound complex 5- $\mathrm{Br}$ was used as starting coordinates and the bromide ligand was deleted. The $6-31 \mathrm{G}(\mathrm{d})$-combo basis set is a hybrid basis set consisting of the Pople 6-31G(d) basis set obtained from the EMSL Basis Set Exchang $\mathrm{e}^{5,6}$ with diffuse functions on all heteroatoms and the ligating carbonyl carbon atoms and the modified basis set (m6-31G) used for iron. ${ }^{7}$ Hessian calculations were performed at the same level of theory as the optimizations. All Hessian calculations showed no imaginary frequencies, and rotations/translations showed energies of less than $5 \mathrm{~cm}^{-1}$. The orbital calculation was performed with the PW91/6-31G(d)-combo and visualized with MacMolPlt. ${ }^{8}$ 


\section{References:}

1. Gaussian 09, Revision A.02, M. J. Frisch, G. W. Trucks, H. B. Schlegel, G. E. Scuseria, M. A. Robb, J. R. Cheeseman, G. Scalmani, V. Barone, G. A. Petersson, H. Nakatsuji, X. Li, M. Caricato, A. Marenich, J. Bloino, B. G. Janesko, R. Gomperts, B. Mennucci, H. P. Hratchian, J. V. Ortiz, A. F. Izmaylov, J. L. Sonnenberg, D. Williams-Young, F. Ding, F. Lipparini, F. Egidi, J. Goings, B. Peng, A. Petrone, T. Henderson, D. Ranasinghe, V. G. Zakrzewski, J. Gao, N. Rega, G. Zheng, W. Liang, M. Hada, M. Ehara, K. Toyota, R. Fukuda, J. Hasegawa, M. Ishida, T. Nakajima, Y. Honda, O. Kitao, H. Nakai, T. Vreven, K. Throssell, J. A. Montgomery, Jr., J. E. Peralta, F. Ogliaro, M. Bearpark, J. J. Heyd, E. Brothers, K. N. Kudin, V. N. Staroverov, T. Keith, R. Kobayashi, J. Normand, K. Raghavachari, A. Rendell, J. C. Burant, S. S. Iyengar, J. Tomasi, M. Cossi, J. M. Millam, M. Klene, C. Adamo, R. Cammi, J. W. Ochterski, R. L. Martin, K. Morokuma, O. Farkas, J. B. Foresman, and D. J. Fox, Gaussian, Inc., Wallingford CT, 2016.

2. Ilic, S.; Kadel, U.P.; Basdogan, Y.; Keith, J.A.; Glusac, K.D. J. Am. Chem. Soc. 2018, 140, 4569.

3. A. A. Granovsky, Firefly version 8, http://www.classic.chem. msu.su/gran/firefly/index.html.

4. (a) Perdew,J. P.;Chevary, J.A.; Vosko,S. H.;Jackson, K.A.; Pederson,M.; R.; Singh, D. J.; Fiolhais, C. Phys. Rev. B: Condens. Matter 1993, 48, 4978.

(b) Perdew, J. P.; Chevary, J. A.;Vosko,S. H.;Jackson, K. A.; Pederson, M. R.; Singh,D. J.; Fiolhais, C. Phys. Rev. B: Condens. Matter 1992, 46, 6671.

5. D. Feller, J. Comput. Chem. 1996, 17, 1571.

6. K. L. Schuchardt, B. T. Didier, T. Elsethagen, L. Sun, V. Gurumoorthi, J. Chase, J. Li and T. L. Windus, J. Chem. Inf. Model. 2007, 47, 1045.

7. A. V. Mitin, J. Baker and P. Pulay, J. Chem. Phys. 2003, 118, 7775.

8. B. M. Bode and M. S. Gordon, J. Mol. Graphics Modell. 1998, 16, 133. 